A R R TII C C U L O O D E I $N$ VES $S$ T I G A C I Ó $N$ $\begin{array}{lllllllllllllllllll}C & I & E & N & T & I & F & I & C & A & Y\end{array}$

TEE C N O L L Ó G I C A

\title{
LOS PROCESOS PEDAGÓGICOS EN LA EDUCACIÓN INICIAL: UNA APUESTA PARA LA CONSTRUCCIÓN DE SUJETOS SOCIALES
}

\author{
THE PEDAGOGICAL PROCESSES IN INITIAL EDUCATION: A \\ PROPOSAL FOR THE CONSTRUCTION OF SOCIAL SUBJECTS
}

\author{
Por: Lina Luz Córdoba Girado* \\ Rosaura Arrieta Flórez**
}

Trabajadora social

gena. Joven investiga-

dora del grupo CTS de

la Universidad de Car-

tagena. E-mail: linacor-

doba7@gmail.com

Recibido: 27 de julio de 2016 - Aprobado: 23 de mayo de 2017

**Economista de la Universidad Externado de Colombia y Magister en Política Social de la Universidad pontificia javeriana. Docente del Instituto de Políticas públicas, Regional y de Gobierno y del Programa de Trabajo Social de la Universidad de Cartagena. Investigadora grupo CTS. E-mail: ipreg2@unicartagena. edu.co

\begin{abstract}
RESUMEN
El presente trabajo describe, analiza y representa los procesos pedagógicos llevados a cabo en diez Centros de Desarrollo Infantil-CDI, modalidad institucional en Cartagena de Indias, con el fin de mostrar la incidencia de estas acciones orientadas a la construcción de sujetos autónomos, reflexivos e investigativos. Se analizaron diez CDI de la ciudad y se encontró que la educación inicial ofrecida en estas instituciones incide de forma directa en el fortalecimiento y desarrollo de capacidades cognitivas, comunicativas, corporales, socioemocionales y estéticas en los niños menores de 5 años que participan de la misma. No obstante, se evidencia una desarticulación entre la educación inicial ofrecida en los CDI y la educación formal, lo cual se constituye en un obstáculo al mejoramiento en la calidad de la educación y al reconocimiento de capacidades potenciadas en los niños mediante la educación inicial.
\end{abstract}

Palabras clave: educación inicial, proceso pedagógico, primera infancia, atención integral, derechos.

\begin{abstract}
The present paper describes, analyzes and represents the pedagogical processes carried out in the Centers of Child Development -CDI (In Spanish), institutional modality of Cartagena, in order to show the incidence of these actions orientated to the construction of autonomous, reflexive and researcher subjects. Ten CDI of the city were analyzed and it was found that the initial education offered in these institutions directly affects the strengthening and development of cognitive, communicative, corporal, socio-emotional and aesthetic capacities in children under 5 years of age who participate Nonetheless, there is a disarticulation between the initial education offered at CDIs and formal education, which constitutes an obstacle to the improvement of the quality of education and to the recognition of enhanced capacities in children through initial education.
\end{abstract}

Keywords: initial education, educational process, early childhood, comprehensive care, rights. 


\section{Introducción}

I onsiderando que los primeros años de vida se convierten en la base sobre los cuales se complejiza el desarrollo humano, la educación inicial como estrategia para la promoción del desarrollo integral de la primera infancia se constituye en la oportunidad para desarrollar y fortalecer capacidades físicas cognitivas, emocionales y estéticas en los niños, las cuales contribuyen de forma directa en el proceso de aprendizaje, socialización y comunicación; y en consecuencia, en su construcción como sujetos de derechos. Sobre esta premisa, la Política Pública Nacional de Primera Infancia "Colombia por la Primera infancia" (2007), plantea que "las investigaciones en diversos campos del conocimiento como la biología, la neurociencia, las ciencias del comportamiento, la sicología del desarrollo, la educación y la economía demuestran que los primeros años de la vida están caracterizados por los desarrollos más importantes que ocurren en la vida de un individuo" (p.2).

Según el CONPES 109 de 2007 por el cual se aprueba y adopta la política publica Colombia por la primera infancia, los primeros años de vida son definitivos para el crecimiento físico y la interconexión neuronal; pues, un niño nace con cerca de 100 billones de células cerebrales (neuronas), pero 95\% de ellas aún no están conectadas entre sí. Después del nacimiento ocurre una extraordinaria interconexión entre las neuronas, para formar las "vías neuronales", las cuales permiten ver, oír, oler, caminar, hablar, pensar. Se trata de un proceso natural, que ocurre de manera muy rápida y casi en su totalidad durante la primera infancia. Es un período privilegiado, en el cual el cerebro es más receptivo para fijar experiencias, y que debe ser aprovechado al máximo. (p. 4 y 5).

Los anteriores fundamentos comprueban la importancia de la educación inicial para el desarrollo integral y potenciamiento de capacidades y habilidades mediante acciones intencionadas con sentido en sí mismas, acompañado de condiciones óptimas para el aprendizaje y disfrute de experiencias significativas. La educación inicial es definida por la Ley 1098 de 2006 -Ley de Infancia y Adolescencia- como un derecho impostergable; por lo tanto, su promoción y garantía son un imperativo inexcusable para los gobiernos, más aún si se pretende contribuir a la paz, el desarrollo humano y la disminución de la pobreza en los territorios del país. En este contexto, se esperaría que las políticas sociales orientaran de manera focalizada esfuerzos, recursos y acciones que permitieran la atención de los niños menores de 5 años en 
Centros de Desarrollo Infantil (CDI); instituciones encargadas de la educación inicial de la primera infancia.

El presente documento presenta los resultados de la investigación realizada durante los años 2015 y 2016 en diez Centros de Desarrollo Integral en Cartagena con el fin de mostrar la incidencia de estas acciones intencionadas en la construcción de sujetos de derechos. En primer lugar se presenta la metodología utilizada, el enfoque y tipo de investigación; posteriormente se realiza una breve explicación de la estrategia de cero a siempre, luego se puntualiza en las implicaciones de la educación inicial ofrecida en los Centros de Desarrollo Infantil, y desde allí, describir los hallazgos de la investigación relacionados a los procesos pedagógicos desarrollados por las docentes con los niños menores de 5 años en los CDI; por último, se presentan algunos obstáculos evidenciados en el proceso.

\section{Metodología}

T a investigación se fundamentó en el enfoque cualitativo y es de tipo descriptiva. Para la recolección de la información referida a los procesos

ــ formativos desarrollados con los niños al interior de los CDI, se recurrió a una revisión documental direccionada al análisis de los lineamientos pedagógicos definidos por la Comisión Intersectorial de Primera Infancia (CIPI), el Ministerio de Educación Nacional y el Instituto de Bienestar Familiar (ICBF) para el desarrollo de capacidades en la primera infancia. Posteriormente se entrevistó a coordinadores pedagógicos y administrativos indagando sobre las dinámicas de la atención al interior de los CDI, los procesos desarrollados, objetivos, herramientas, seguimientos, componentes de atención, entre otros aspectos; y se dialogó con las agentes pedagógicas, ahondando en las metodologías, técnicas, instrumentos y estrategias utilizadas para el potenciamiento y fortalecimiento de capacidades en los niños y su articulación con las pilares y dimensiones del desarrollo infantil propuestas para ello. Los CDI analizados fueron: Fundación Granitos de Mostaza, Fundación Actuar por Bolívar, Colegio Educativo los Ángeles, Corporación Paulo Freire, Asociación Fomento al Desarrollo Social (FODES), Fundación HIJOS DE BOLÍVAR, Corporación Educativa Colegio Gran Colombia, Fundación Preservar por Colombia y Fundación Granitos de Paz. La información recolectada fue sistematizada y analizada utilizando el software Atlas ti, las categorías de análisis se enmarcaron en las estrategias, instrumentos y actividades desarrolladas por las agentes en los procesos pedagógicos, las dimensiones y pilares del desarrollo infantil, la potenciación de capacidades físicas, cognitivas, 
emocionales y estéticas, la construcción de sujeto de derecho y la articulación de la educación inicial con la educación preescolar.

\section{La educación inicial en la estrategia de cero a siempre}

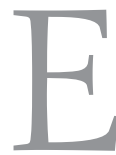

n Colombia, La Política Pública Nacional de Primera Infancia "Colombia por la Primera Infancia" fue resultado de un proceso de construcción con significativas transformaciones que contribuyó al reconocimiento de las niñas y los niños como sujetos de derecho y la obligatoriedad del Estado en garantizar, proteger y restablecer en corresponsabilidad con la sociedad y la familia los derechos impostergables de la primera infancia.

Esta política pública nacional, fue adoptada mediante el Conpes 109 de 2007, y dentro de los hechos que posibilitaron su adopción se pueden mencionar la Convención Internacional sobre los Derechos del Niño, la aprobación de la Convención Internacional sobre los Derechos del Niño por el Congreso de la República de Colombia (Ley 12 de 1991), su incorporación en la Constitución Política de 1991 con el artículo 44 y la promulgación de la Ley 1098 de 2006 por la cual se expide el Código de la Infancia y la Adolescencia. En la actualidad, esta política pública nacional se ejecuta mediante la estrategia de gobierno de cero a siempre.

De cero a siempre, es la estrategia nacional de atención integral a la primera infancia que busca aunar los esfuerzos de los sectores público y privado, de las organizaciones de la sociedad civil y de la cooperación internacional en favor de la primera infancia de Colombia. La Consejería Presidencial para la Primera Infancia busca transformar la manera sectorizada en la que se han suministrado los servicios de atención a la primera infancia creando la estrategia nacional de cero a siempre, que reúne políticas, programas, proyectos, acciones y servicios dirigidos a la primera infancia, con el fin prestar una verdadera atención integral que haga efectivo el ejercicio de los derechos de los niños y las niñas entre cero y cinco años de edad. (De cero a siempre, 2016, párr. 1) En síntesis, la estrategia de gobierno De cero a siempre, fue creada en el año 2011, gracias a los trabajos de la Comisión Intersectorial de Atención Integral a la Primera Infancia en marco del plan de desarrollo Prosperidad para Todos. Sin embargo, para el año 2016, como parte del compromiso político por la primera infancia de Colombia, se adopta la Ley 1804 del 2 de agosto, por la cual establece la política de Estado para el desarrollo integral de la primera infancia De cero a siempre y se dictan otras disposiciones. Cuyo propósito radica en "fortalecer el 
marco institucional para el reconocimiento, la protección y la garantía de los derechos de las mujeres gestantes y de los niños y las niñas de cero a seis años de edad, así como la materialización del Estado Social de Derecho" (Ley 1804 de 2016, pág. 1).

La estrategia de cero a siempre, está conformada por cinco ejes estructurales: i) Cuidado y crianza, ii) Salud, nutrición y alimentación, iii) Educación inicial, iv) Recreación y v) Ejercicio de la ciudadanía. Dentro del eje de educación inicial, la Comisión Intersectorial de Atención Integral a la Primera Infancia ha definido las modalidades de atención así: Centros de Desarrollo Infantil (CDI) y Modalidades familiares. El propósito de estas modalidades es lograr coherencia al reorganizar los servicios y unificar criterios entre los sectores al momento de garantizar las diversas atenciones que requieren los niños en pro a su desarrollo integral. Por otra parte, la modalidad comunitaria, es una modalidad de atención no integral (cursiva nuestra) que corresponde a Hogares Comunitarios de Bienestar (HCB), ha sido el programa tradicional definido y ofertado por el ICBF, el cual es operado por las madres comunitarias en sus residencias, sin apoyo psicosocial. En el año 2010, con la implementación de la estrategia De cero a siempre se empiezan a transitar de los Hogares Comunitarios de Bienestar (HCB) a los Centros de Desarrollo Infantil (CDI) para asegurar el desarrollo integral de la población de primera infancia en Colombia, mediante atención y acompañamiento cualificado en ambientes seguros y protectores, en el reconocimiento de los niños como sujetos de derechos y protagonista de su propio desarrollo. Cabe señalar, que los Centros de Desarrollo Infantil (CDI) también corresponden a la modalidad de servicio de atención institucional del ICBF.

Según el Ministerio de Educación Nacional-MEN (2012) en los CDI se planean y desarrollan acciones dirigidas a materializar los derechos a la educación inicial, cuidado, salud, nutrición, protección, participación y acceso a bienes culturales, a través de una intervención en las dimensiones del Desarrollo Infantil Temprano, atenciones que se han definido como inherentes a la concepción de desarrollo integral. En este sentido, los equipos profesionales que acompañan y desarrollan el proceso deben ser interdisciplinarios con el fin de orientar la acción en la comprensión holística de la atención. Los CDI operan a través de organizaciones comunitarias, sociales y educativas, quienes desarrollan procesos de mejoramiento continuo debido a la definición de componentes de calidad que son evaluados por medio de estándares 
definidos en el Sistema de Gestión de Calidad para los CDI, estos son: i) Proceso administrativo y de gestión, ii) Ambientes educativos y protectores, iii) Proceso pedagógico, iv) Salud y nutrición, v) Talento humano, y vi) Familia comunidad y redes.

\section{Hallazgos en los procesos pedagógicos de los CDI en Cartagena, 2015}

\section{Proceso pedagógico para el fortalecimiento de capacidades en la promoción del desarrollo integral en la primera infancia}

- a educación inicial además de ser un eje estructural de la atención integral de la primera infancia definida por la estrategia De cero a siempre, es un derecho impostergable según la Ley de Infancia y Adolescencia, que tiene por objetivo el cuidado y acompañamiento del crecimiento y desarrollo de los niños mediante la creación de ambientes de socialización seguros y sanos" (Conpes 109, p 23).

Los procesos pedagógicos desarrollados con los niños menores de 5 años en el marco de la educación inicial, deben estar direccionados a una educación emancipadora, en la co-construcción de sujetos críticos, propositivos, autónomos y participativos, ampliando las posibilidades en los niños de elegir libremente el tipo de vida que desean en su realización como personas. Para asegurar la efectividad de los procesos pedagógicos desarrollados al interior de los CDI, estos deben abordar desde una perspectiva holística e integral las dimensiones del desarrollo humano, los pilares definidos como actividades rectoras en la infancia mediadas por estrategias pedagógicas que permiten organizar flexiblemente el trabajo pedagógico, tal articulación posibilita el desarrollo y potenciación de capacidades en los niños como autores de aprendizajes, conocimientos, realidades y significados.

Se definieron cinco dimensiones del desarrollo humano: "comunicativa, corporal, persona social, cognitiva y estética" (CIPI, 2013, p. 40), las cuales están estrictamente relacionadas, por ende, no pueden ser entendidas como "compartimentos separados, sino como parte de un todo, coexistiendo en cada una de ellas una serie de componentes" (Amary Abello 2004, p.33 como se citó en Galindo, 2014). En el reconocimiento de las particularidades de cada dimensión en relación con las demás se puede entender la integralidad del desarrollo infantil. Estas no se desarrollan por sí solas, ni de forma jerárquica, de allí la importancia de las intervenciones de las agentes pedagógicas para potenciarlas en su máxima expresión. 
La relación existente entre las dimensiones del desarrollo y los pilares radica en que mediante la promoción del juego, la literatura, el arte y la exploración al medio se potencian las dimensiones cognitivas, comunicativa, corporal, persona social y estética en los niños de forma sistemática, intencionada y autónoma, materializando el desarrollo de capacidades. "Al jugar al papá y a la mamá, el niño y la niña están desarrollando la capacidad simbólica y en este sentido, la dimensión cognitiva; el lenguaje y por ende la dimensión comunicativa (...) asimismo la dimensión personal social, pues están aprendiendo a convivir y a relacionarse con otros" (Comisión Intersectorial para la Primera Infancia, 2012 p.35).

\section{Estrategias pedagógicas en la educación inicial}

Las estrategias pedagógicas son las "formas de trabajo organizadas que se han construido desde ciertas posturas sobre la enseñanza y el aprendizaje (...) que posibilitan planear y organizar momentos, ambientes, interacciones y experiencias que llenan de sentido el quehacer cotidiano de una maestra o agente educativo con un grupo de niños y niñas" (Comisión Intersectorial de Primera Infancia-CIPI,2012, p.123). Estas estrategias se caracterizan por ser intencionadas y flexibles, pues solo sirven para orientar el trabajo pedagógico, este último, debe ir direccionado a fortalecer las dimensiones del desarrollo infantil que son i) "cognitiva ii) corporal iii) persona social iv) estética y v) comunicativa" (CIPI, 2012, p.33), las cuales son traducidas en capacidades y habilidades en los niños mediante las actividades propias de la infancia: el juego, arte, literatura y exploración del medio definidas en marco de la atención integral-estrategia De cero a siempre.

Estas estrategias pedagógicas están fundamentadas en la teoría constructivista y el construccionismo social; la primera dirigida a "generar situaciones significativas para que los niños potencien su desarrollo integral desde las interacciones con otros niños, con el medio y con los demás, utilizando el juego, el arte, la literatura y la exploración del medio"(ICBF,2103, p.20). La segunda, encaminada a la construcción de conocimientos a través del contacto con el entorno social y cultural, considerando "que las personas llegan a describir el mundo social o dar cuenta del mundo donde viven, a partir de una construcción de conocimiento que se da en un determinado contexto cultural e histórico" (ICBF,2103 p.45) desde este enfatiza en el papel del entorno social y cultural que les rodea, desarrollando un fuerte trabajo orientado a incluir el entorno en el ambiente educativo y abrir sus prácticas pedagógicas hacia la comunidad. Procesos fundamentados en 
diferentes modelos teóricos que posibilitan el trabajo autónomo, flexible y significativo.

Las estrategias propuestas por la CIPI son: proyecto pedagógico, proyecto de aula, asambleas y rincones pedagógicos. Las actividades pedagógicas desarrolladas con los niños menores de cinco años al interior de los Centros de Desarrollo Integral de Cartagena, se encuentran en el proyecto pedagógico de la Entidad Administradora del Servicio como parte del PAI -Plan de Atención Integral (eje de la planeación y articulador de los procesos de atención en los Centros de Desarrollo Infantil). Los proyectos pedagógicos institucionales se formulan a raíz de las necesidades de los niños identificadas mediante el diagnóstico social del entorno donde se desenvuelven, con participación directa de las familias y comunidad. Su objetivo es intentar dar soluciones a las problemáticas reconocidas. Asimismo, el proceso pedagógico direccionará actividades socializadoras con los niños relacionados a la resolución pacífica de conflictos, buen trato, convivencia familiar, derecho sobre el cuerpo, entre otras experiencias significativas que ayudarán a la trasformación de la realidad. Del mismo modo, los proyectos de aula se constituyen en subproyectos del proyecto pedagógico institucional dirigidos por cada docente de los ambientes pedagógicos, en coherencia a los objetivos propuestos en el mismo.

"Acá trabajamos por proyectos, de acuerdo a la necesidad de los niños. Se les pregunta al niño que quiere conocer, creamos el proyecto y le damos a conocer el mismo". (Entrevistada 9. Agente pedagógica, 2015)

Otra estrategia utilizada en los CDI es la asamblea, la cual consiste en adaptar un espacio dentro del ambiente pedagógico, para el encuentro interpersonal entre niños y la docente, desde este escenario se dialoga sobre asuntos de la vida cotidiana, dando apertura a la socialización de las actividades a desarrollar durante la jornada. Esta estrategia tiene como objetivo principal premiar la participación de los niños en las actividades pedagógicas, reconocidos como sujetos propositivos y autores del conocimiento.

"Se les da la bienvenida, se le pregunta que hicieron antes de llegar acá, el tema que vimos el día anterior y les decimos que trabajaremos hoy". (Entrevistada 12. Agente pedagógica 2015)

Este primer momento se le llama bienvenida, la cual consiste en dar recibimiento humano a cada niño cuando llega a la institución, conversando 
temas de la vida cotidiana, a la vez, se indaga sobre su estado emocional, convirtiéndose en un espacio de conversación y diálogo caracterizado por la ausencia de imposiciones, que permite la expresión libre de los niños, de sus experiencias, sentimientos y expectativas. Luego de este momento, se procede a la planeación y socialización de las actividades para la jornada. Las asambleas en los CDI de la ciudad se realizan todos los lunes y el primer día de cada mes.

"Semanalmente los lunes le damos a conocer los temas que se darán en la semana, eso lo hacemos los lunes y el primer día del mes". (Entrevistada 8. Agente pedagógica, 2015)

A partir de la información de las entrevistadas se encontró que esta estrategia - que permite la participación activa de los niños- posibilita el desarrollo y enriquecimiento de las dimensiones comunicativas, cognitivas, personal y social en los niños, teniendo incidencia en el desarrollo artístico y corporal.

"El niño aprende mucho cuando estamos en la asamblea, cuando está al lado del compañerito, cuando da su opinión. (...) Cuando pregunta desarrollamos la capacidad de pensar, comunicarse". (Entrevistada 5. Agente pedagógica, 2015)

En conclusión, el uso de asamblea como estrategia en el proceso pedagógico hace posible el desarrollo de las dimensiones del desarrollo infantil en los niños que participan en ella, pues las actividades desarrolladas allí, permiten el reconocimiento del sujeto en sus emociones, experiencias, conocimientos, necesidades y derechos, haciéndole sentir valiosos en la escucha de sus opiniones y aportes; lo que fortalece la estima propia y da significado a la existencia de los otros, mediante la construcción del valor de la amistad, la solidaridad, el respeto y la confianza. En este sentido, los niños van adquiriendo conciencia de los derechos que poseen, de expresar libremente lo que sienten y piensan, en proponer, y ser respetado por sus singularidades. Además mediante las interacciones dadas en la asamblea, los niños empiezan a comprender al "otro" desde sus particularidades, y a través del respeto de las normas entienden los principios y valores culturales necesarios para la convivencia con el otro.

Otra estrategia pedagógica utilizada en los CDI son los rincones, los cuales consisten en ambientar y adecuar el espacio pedagógico de acuerdo a las 
actividades rectoras en la infancia: juego, literatura, arte y exploración del medio, cada uno ubicado en lugares estratégicos dentro del aula.

"Los rincones los establecemos dentro de los ambientes de aprendizajes, que son las aulas, dotados de elementos reciclables necesarios para potencializar de forma simultánea las dimensiones del desarrollo infantil. Estamos trabajando las dimensiones comunicativa, corporal, cognitiva, se trabajan estas dimensiones al mismo tiempo. En esta medida, las actividades desarrolladas en los rincones son planeadas y organizada en un planeador a partir de las necesidades e inquietudes identificadas durante el proceso pedagógico en los niños". (Entrevistada 1. Agente pedagógica, 2015)

Asimismo, las actividades a desarrollar mediante los rincones son creadas a partir de las necesidades e inquietudes identificadas por la docente durante el proceso pedagógico en los niños:

"Se trabaja según las inquietudes que tiene el niño, se crean las estrategias, la docente decide cuantos días trabajará ese tema (...) La docente analiza qué habla, qué hace, qué quiere saber, a partir de eso se planean los temas, según las inquietudes, si el niño viene hablando sobre la flor, ese puede ser un tema para el día siguiente y se crean las estrategias, la docente lo maneja durante un día, dos de acuerdo a lo que se dé". (Entrevistada 8. Agente pedagógica, 2015)

Los rincones en los CDI de la ciudad, se organizan a partir de las actividades propias de la infancia, los cuales llevan el nombre de las mismas, es decir; rincón de juego, de literatura, de arte y de exploración al medio, y tienen por objetivo el desarrollo simultáneo de capacidades en las dimensiones cognitiva, corporal, estética, comunicativa y personal social en los niños con el propósito de asegurar un desarrollo integral.

"Estamos trabajando las dimensiones comunicativa, corporal, cognitiva, se trabajan estas dimensiones al mismo tiempo". (Entrevistada 3. Agente pedagógica, 2015)

Los rincones, se caracterizan por ser flexibles, autónomos, diferenciados y lúdicos, los cuales permiten al niño aprender según sus necesidades y singularidades; estos se encuentran dotados de elementos reciclables utilizados para potencializar capacidades en los niños. 
Los procesos pedagógicos de los rincones en los Centros de Desarrollo Infantil en la ciudad, se trabajan mediante "momentos", durante estos, los niños se rotan por los diferentes espacios intencionados según sus intereses y desarrollan allí las actividades propuestas para ese día. La agente pedagógica solo guía el proceso de acuerdo a lo anteriormente planeado y "provoca" la acción de los niños y las niñas, y son ellos y ellas quienes deciden qué hacer y cómo hacerlo:

"Ellos escogen lo que quieren hacer, de acuerdo al tema por las "provocaciones". (Entrevistada 5, Agente pedagógica 2015)

El juego, al igual que las demás actividades rectoras, transversaliza todos los procesos pedagógicos desarrollados en los diferentes rincones.

“Todo lo hacemos jugando". (Entrevistada 10. Agente pedagógica 2015)

El juego es una actividad natural para los niños, mediante el aprenden a estar en contacto con el mundo, y es en esa interacción con el entorno, con el otro y con los objetos que "descubren sus habilidades corporales y las características de las cosas" (Ministerio de Educación Nacional, 2015) constituyéndose en la oportunidad para descubrir, conocer, imaginar, crear, representar, pensar, identificarse y experimentar.

Asimismo, el juego "brinda la posibilidad de movilizar estructuras de pensamiento, al preguntarse "qué puedo hacer con este objeto", y es a partir de ello que los participantes desarrollan su capacidad de observar, de investigar, de asombrarse, de resignificar los objetos y los ambientes y de crear estrategias" (Ministerio de Educación Nacional, 2015). El juego desde la perspectiva social, permite la socialización de los niños, ya que es reflejo de la cultura y la sociedad, "en el se representan las construcciones y desarrollos de un contexto" ((Ministerio de Educación Nacional, 2015), cuando los niños juegan asignan significado a la vida social, por lo tanto representan la "cultura en la que crecen y se desenvuelven; la riqueza de ver el juego desde esta perspectiva permite aproximarse a su realidad y a la manera en que la asumen y la transforman" (Ministerio de Educación Nacional, 2015, p.15)

"Con el juego las capacidades que desarrollan es que sepan acatar reglas, aprendan a respetar turnos, a convivir, compartir". (Entrevistada 12. Agente pedagógica 2015) 
Con el juego el niño aprende a convivir pacíficamente con el otro, respetarlo, valóralo y estimarlo, propiciándose el desarrollo de la dimensión personal social. A través de la interacción con los demás; empiezan a comprender la importancia de las normas en la convivencia con el otro, al mismo tiempo, se desarrollándose de forma simultanea las dimensiones del desarrollo infantil en el contacto con los otros y el entorno.

"Con el juego se desarrolla la capacidad cognitiva, comunicativa, corporal, socio afectiva y otras más". (Entrevistada 1. Agente pedagógica 2015)

En los Centros de Desarrollo del Distrito, los rincones se ambientan según las dimensiones que se busca potenciar en los niños. Estos se clasifican así: Rincón juego de roles: en este espacio los niños tienen la libertad de jugar a ser el "otro" entendido desde la cotidianidad, posibilitando el intercambio de roles y el aprendizaje de la cultura:

"Los niños asumen roles, construyen" "juegan a la mamá, al papaá, al doctor". (Entrevistada 8. Agente pedagógica 2015)

En este sentido, representan la realidad según lo que viven en familia, comunidad y escuela. Este rincón es caracterizado por la dotación de materiales indispensables para promover la creatividad, siendo los juguetes, disfraces, entre otros los instrumentos facilitadores de la misma.

"Adquieren habilidades comunicativas, corporales, trabajamos con las inteligencias". (Entrevistada 8. Agente pedagógica 2015)

Mediante el juego de roles los niños aprenden a reconocer y asumir los roles establecidos por la sociedad, aprenden la cultura, normas, dinámicas y las formas de estar y ser en ella, a relacionarse con los demás, permitiendo la construcción de la identidad, el fortalecimiento de la autoestima y autonomía. El desarrollo de todos estos conocimientos queda almacenado en la estructura mental de los niños por los significados asignados a las experiencias vividas en la interacción social, viabilizando que se complejice de la dimensión cognitiva.

Rincón de la construcción: en este espacio los niños cuentan con materiales reciclables con los cuales construyen objetos. Este trabajo grupal se caracteriza por la interacción permanente entre sus integrantes en la imaginación de ideas creativas. Además, en este rincón se cuenta 
con rompecabezas, juegos de lógica, utilizados para la potenciación de capacidades cognitivas, creativas, asociación:

"Los niños cuando arman y desarman, se ve la creatividad, el ingenio, lo que ellos mismos hagan" "tenemos rompe cabezas, arma todo". "Trabajamos la lógica, con los arma todo, manejan forma y colores". (Entrevistada 7. Agente pedagógica 2015)

En esta medida, se estimula el desarrollo del ingenio, la lógica, motricidad fina y al estar en interacción con los demás compañeros aprenden el valor de la amistad, solidaridad fortaleciendo su autoestima y autonomía.

Rincón de juegos: Es un espacio para desarrollar juegos intencionales libres y tradicionales con los niños. Mediante el juego se incide en el desarrollo de todas las diferentes dimensiones; en la interacción con los otros, el niño aprende la cultura, potencializa capacidades físicas y cognitivas, afianza las emociones consigo mismo y hacia los demás, aprende a ser crítico, creativo, a tener control de los movimientos, aportando a la construcción de la personalidad, el reconocimiento del otro y el desarrollo de la autonomía.

"Con el juego se desarrolla la capacidad cognitiva, comunicativa, corporal, socio afectiva, aprenden los niños a acatar reglas, aprenden a respetar turnos, a convivir, compartir, acá todo lo hacemos jugando". (Entrevistada 13, agente pedagógica 2015)

Rincón de literatura: espacio direccionado a desarrollar principalmente las dimensiones cognitivas y comunicativas en los niños, en este, no se les enseña a leer la letra, mediante lo que tradicionalmente se conoce como hacer planas. Se les enseña a "leer" pero como la capacidad para interpretar lo que observan y explorar mundos simbólicos.

"En el rincón de literatura se maneja la lectura, se proyecta videos, con esto se motiva el interés de los niños por la lectura, a ellos se les presenta imágenes en los libros y van creando su cuento, no saben leer pero van interpretando con cuentos de imagen, son libres de decir que pasa, según la imagen". (Entrevistada 4. Agente pedagógica 2015)

El niño en este rincón, desarrolla el interés temprano en la lectura, capacidad de imaginar, indagar, investigar, interpelar, inventar mundos simbólicos, expresar libremente su opinión y escuchar con respeto al otro. 
"Con la literatura se desarrolla la capacidad cognitiva uno les muestra y ellos preguntan, ellos captan" (Entrevistada 3. Agente pedagógica 2015)

Aportando al mismo tiempo a la generación de capacidades comunicativas en el uso del lenguaje y el aprendizaje de nuevas palabras, con las cuales enriquecen su vocabulario.

"Los niños se aprenden cuentos, trabalenguas, poesías, rondas". (Entrevistada 9. Agente pedagógica 2015)

Asimismo, durante este momento, se estimula la creatividad, la imaginación, argumentación, investigación, normas de convivencia, entre otras capacidades en los niños.

"Con la lectura generamos que los niños aprendan a pensar, a argumentar, cuando entramos a la biblioteca, aprenden a alzar la mano para dar su opinión, no tienen miedo de levantar la mano y opinar, aprenden el libre desarrollo de su personalidad, a ser críticos, a investigar". (Entrevistada 14. Agente pedagógica 2015)

Rincón de arte: este espacio está dotado de elementos artísticos para que los niños puedan explorar el arte; en este rincón se le da prioridad al libre desarrollo de la creatividad e imaginación de los niños.

"Acá ellos pueden rasgar, pintar, colorear, hay arte dramatizado, hay música, exploran si les gusta la música, por medio de la música interactúan, afinan, cantan, hacen la ronda, mejoran su vocabulario con las canciones" "hacemos manualidades con materiales reciclables y decoramos sus creaciones". (Entrevistada 10. Agente pedagógica 2015)

Por medio del arte los niños expresan lo que sienten y representan la realidad, permitiendo desarrollar la creatividad, sensibilidad, expresividad y el sentido estético. El arte "posibilita integrar las experiencias de vida con lo que sucede tanto en el entorno educativo como en los otros espacios en los que transcurre la vida de los niños. De esta manera, las experiencias artísticas se convierten en formas orgánicas y vitales de habitar el mundo y contribuir a evidenciar, por medio de diversas formas de comunicación y expresión, la necesidad simbólica que hace disfrutar la vida, contemplarla, transformarla y llenarla de sentido" (Ministerio de Educación Nacional, 2014). 
Rincón de exploración al medio: mediante la exploración los niños tienen acercamiento directo o indirecto con el medio que les rodea, "identifican que existen objetos naturales y otros que son construidos por el ser humano; se acercan a los fenómenos físicos y naturales; reconocen las diferentes formas de relacionarse entre unas y otras personas, construyen hipótesis sobre el funcionamiento de la naturaleza o de las cosas, y se apropian de su cultura" (Ministerio de Educación Nacional, 2015). Esto significa que los niños en contacto con el mundo exterior, empiezan a entender lo social y lo natural en permanente interacción, reconocen la existencia de los objetos y fenómenos. La docente debe orientar intencionalmente la actividad para el conocimiento y comprensión de lo que les rodea.

"En la huerta escolar también hacemos proyectos, por ejemplo, cómo crecen las plantas, qué necesitan para vivir, cuál es el proceso de crecimiento, estimulando en los niños la capacidad de pensar, buscamos el porqué de las cosas, no tratamos de ser nosotras las que le demos las respuestas sino que ellos mismos las vayan construyendo por lo que nosotras enseñando, le mostramos, encontrando. Para la exploración del medio les traigo por ejemplo frutas para enseñarle cuales son los alimentos que nos dan energía". (Entrevistada 10. Agente pedagógica 2015)

Mediante la exploración al medio el niño no solo puede observar lo que les rodea, sino también manipular, experimentar y expresar las sensaciones producidas en contacto con la naturaleza. Se fortalece la curiosidad, como la capacidad de querer encontrar explicaciones, comprender y conocer el mundo, desarrollándose la reflexión, sensibilidad, indagación y descripción.

En conclusión, las estrategias utilizadas por las docentes en los procesos pedagógicos con los niños en los Centros de Desarrollo Infantil de Cartagena, contribuyen de forma directa al desarrollo de capacidades comunicativas, cognitivas, emocionales, sociales, corporales y estéticas en los niños, en la formación de seres sociales, críticos, autónomos, reflexivos, investigadores, propositivos, innovadores, creativos, sensibles en últimas a su construcción como sujeto de derecho:

"Los testimonios que tenemos, es que los niños que egresan de acá, son muy buenos, de que a pesar que no se les enseña a escribir, pues si se les enseña a tener un pensamiento crítico, holístico, que puedan argumentar, describir y tienen la capacidad de hablar, argumentar y van mucho 
más fortalecidos en la parte emocional, pues nos centramos en suplir la necesidad del niño como sujeto de derecho, de brindarle lo que realmente el necesita, no lo que decidamos". (Entrevistada 8. Agente pedagógica 2015)

Estos procesos flexibles que premian la participación y protagonismo de los niños en la construcción de su propio aprendizaje, posibilitan el disfrute pleno de experiencias verdaderamente significativas.

Los procesos pedagógicos desarrollados en los Centros de Desarrollo Infantil, inciden en la formación de niños con capacidad de "convivir con otros seres humanos, de establecer vínculos afectivos con pares y adultos significativos, diferentes a los de su familia, a relacionarse con el ambiente natural, social y cultural; a conocerse, a ser más autónomos, a desarrollar confianza en sí mismos, a ser cuidados y a cuidar a los demás, a sentirse seguros, partícipes, escuchados, reconocidos; a hacer y hacerse preguntas, a indagar y formular explicaciones propias sobre el mundo en el que viven, a descubrir diferentes formas de expresión, a descifrar las lógicas en las que se mueve la vida, a solucionar problemas cotidianos, a sorprenderse de las posibilidades de movimiento que ofrece su cuerpo, a apropiarse y hacer suyos hábitos de vida saludable, a enriquecer su lenguaje y construir su identidad en relación con su familia, su comunidad, su cultura, su territorio y su país" (Ministerio de Educación Nacional, 2015).

Por ende, la garantía de la educación inicial en los territorios constituye en el imperativo para la administración local, primero, porque es un derecho impostergable que debe ser protegido, ya que está respaldado por normativas internacionales y nacionales; segundo, porque es la oportunidad para que los niños desarrollen de forma integral todas sus dimensiones; tercero, porque establece en la estrategia para promover la igualdad, paz y el desarrollo humano en el territorio cartagenero; cuarto, para lograr que los niños de la ciudad puedan vivir felices en los espacios donde transcurre su vida cotidiana; y quinto, para formar agentes transformadores de la realidad social en la que está inmerso, en las diferentes formas creativas e innovadoras de resignificarla. De este modo, la educación inicial en tanto proceso pedagógico deja una "huella imborrable para toda la vida" (Ministerio de Educación Nacional, 2015) en cada niño que participa de ella; pues gracias al disfrute de experiencias significativas fundamentadas en el juego, el arte, la literatura y la exploración del medio, se potencializa el desarrollo de la primera infancia. 
No obstante lo anterior, estos desarrollos no se articulan en todos los casos con los niveles de educación formal, esta última, caracterizada por la relación lineal docente-estudiante, evaluación del conocimiento, organización de las áreas y competencias específicas del saber, contenidos y metodologías preestablecidas, espacios formales para los aprendizajes y tiempos definidos según temáticas. Todos estos elementos diferentes a los procesos pedagógicos desarrollados en la educación inicial en los CDI en donde se ubica al niño como autogestor de conocimientos y aprendizajes, siendo la docente quien solo orienta de forma intencionada las actividades sin ninguna imposición. Esta estrategia constituye la relación docente-niño en una relación de doble vía, en la cual se generan experiencias significativas a los niños con aprendizajes no segmentados por áreas del conocimiento ni mucho menos evaluados por competencias específicas; en últimas, con la educación inicial se pretende responder la necesidad de aprendizajes del niño de acuerdo a su edad, ritmo y particularidades.

"En primaria todo es diferente, las evaluaciones, las actividades, como utilizan el tiempo y para el niño se convierte en un cambio brusco, siempre tiende a ver un desequilibrio allí, nosotras tratamos de desarrollar las bases para que los niños aprehendan a asociar, hagan conexión, sin recurrir estrictamente a la enseñanza de educación formal. Clasificar, mencionar, identificar, no como la educación formal. Entonces en esto hay un vacío que el ministerio debe arreglársela, porque estos niños que vienen con un ritmo donde él elige lo que va a hacer y luego en el colegio deba hacer lo que le mande la profesora, es un cambio brusco. La educación formal no admite esta dinámica, sobre todo en el comportamiento. Esto va hacer un poquito difícil para los niños". (Entrevistada 5. Coordinadora pedagógica 2015)

En este panorama de desencuentro y desarticulación entre la educación inicial y la formal debido a las diferentes dinámicas de las mismas para el aporte de conocimientos a los niños, el tránsito de una etapa a otra ocasiona interrupciones, frustraciones y cambios bruscos en el proceso escolar de los niños, con las evidentes consecuencias de los bajos niveles de aprestamiento para el aprendizaje integral y de desarrollo de potencialidades que permitan la continuidad en el ciclo de formación. En esta medida, la transición de los niños de la educación inicial a la formal se constituye en un factor importante para asegurar la continuidad del desarrollo y óptimos aprendizajes de acuerdo a su edad; tal articulación debe comprometer la integración de la educación inicial con la preescolar y esta, a la vez con la primaria como eslabones de un 
único proceso, permitiendo el reconocimiento de capacidades, conocimientos, competencias, aprendizajes desarrollados en etapas anteriores y necesariamente potenciados en las subsiguientes. Entonces, ¿Cómo lograr que la educación inicial y la educación formal sean caras de una misma moneda? ¿Cómo lograr una articulación efectiva? ¿Qué elementos deben ser tenidos en cuenta para la articulación de estos procesos?

La transición educativa es entendida como el "cambio que efectúan los niños desde una fase de la educación a otra, en el que se enfrentan a desafíos desde el punto de vista de las relaciones sociales, el estilo de enseñanza, el ambiente, el espacio, el tiempo, los contextos de aprendizaje y el aprendizaje mismo, haciendo de este proceso algo intenso y con demandas crecientes" (Fabián, 2006, p.140, como se citó en Argos, 2011).

Por ello la importancia de fortalecer los procesos desarrollados en las etapas anteriores, pues son la base que complejizarán los conocimientos y aprendizajes posteriores; las estrategias pedagógicas articuladoras deberán garantizar un desarrollo progresivo, continuo e integral, que respete el ritmo y las características del niño de acuerdo a su edad y desarrollo, con el objetivo de mitigar las posibles frustraciones y desequilibrios en el tránsito de una etapa a otra, posibilitando la adaptación fácil y natural al nuevo contexto, espacio y dinámica escolar. Significa lo anterior, que la educación en tanto formación en las edades iniciales es la plataforma que deberá fortalecerse desde una articulación sólida entre educación inicial y la formal, para el logro de una educación pertinente, inclusiva y de contribución al desarrollo humano y a la construcción de mejores procesos de convivencia social.

\section{Conclusiones y recomendaciones}

i se parte del supuesto referido a la educación inicial como un derecho impostergable de la primera infancia, que establece la oportunidad para
desarrollar y promover capacidades físicas, cognitivas, emocionales y sociales en los niños y niñas, se hace necesario pensar en estrategias pedagógicas que permitan articular de forma integral los procesos desarrollado desde la educación inicial con la formal, para contribuir y dar continuidad al desarrollo de capacidades físicas, cognitivas, emocionales y sociales en los niños, en pro a la construcción permanente de sujetos de derechos. En este contexto, con el fin de mejorar la calidad educativa en el país, y que desde esta, se promueva el desarrollo integral de los niños, se deben generar procesos de articulación efectiva entre la educación inicial, preescolar y primaria que 
asegure la continuidad del mismo; con estrategias pedagógicas construidas entre los diferentes entes implicados en la educación de la infancia.

Algunas estrategias pedagógicas propuestas desde la investigación relacionadas al logro de articulaciones entre los CDI con las instituciones educativas para dar continuidad a los procesos desarrollados desde este escenario, están encaminadas a la promoción de espacios de encuentro de los niños de los CDI con las dinámicas del colegio, con las denominadas visitas escolares, para el logro de socialización con el ambiente, actividades, compañeros y maestros. Además, propender por mantener en el grado preescolar la transverzalización del juego, la literatura, el arte y la exploración al medio en las actividades desarrolladas en este grado sin dejar a lado el fortalecimiento de capacidades o competencias propias de la educación preescolar. Así mismo, propiciar ambientes enriquecidos para el aprendizaje, y sobre todo preparar a las docentes sobre las diferentes formas de actuación pedagógica, las características del desarrollo en etapas inicial y posterior y sobre los procesos formativos desarrollados con los niños; propuesta que podría ser pertinente para la integración de preescolar con el grado de primero de primaria del sistema educativo colombiano. En esta medida, el logro de una articulación efectiva entre estas tres esferas se convierte en un reto para los docentes, no solo de aquellos que orientan al niño, sino también de aquellos que potenciarán en otros niveles su desarrollo.

Las anteriores propuestas se constituyen en sugerencias que desde el campo de la investigación social se aporta para el mejoramiento de la calidad educativa y la promoción del desarrollo integral de los niños en los primero años de vida. Sin embargo, desde las facultades de educación en las universidades del país se deben generar espacios de diálogo y debate tendientes a la construcción de estrategias que coadyuven a procesos de tránsitos armónicos y flexibles de la población infantil a los niveles de educación formal. Asimismo, se debe pensar en los elementos posibilitadores de la integración no solo de la educación inicial con la preescolar, sino también de esta última con primero de primaria. Desde aquí habría que plantearse estrategias que posibiliten el reconocimiento del niño desde sus necesidades y particularidades, es decir, que ese mismo niño que egresa de los CDI a preescolar y de preescolar a primero de primaria "sigue siendo el mismo niño, con interés de jugar, pintar, explorar, trabajar con material variado, de diversos colores, formas y tamaños, un salón de clase con un ambiente que lo invite a experimentar" (Durán, 2009). En últimas, tal articulación debe estar encaminada al libre desarrollo del sujeto, como 
agente autoconstructor de conocimientos, significados y procesos personales con cambios paulatinos que posibiliten complejizar capacidades previamente desarrolladas; articulación de carácter continuo que permita unir eslabones indistintamente de sus particularidades y exigencias y permita entender al niño desde su desarrollo y características.

\section{REFERENCIAS BIBLIOGRÁFICAS}

Argos, Ezquerra \& Castro (2011). "Metáforas de la transición: la relación entre la escuela infantil y la escuela primaria y la perspectiva de futuros docentes de educación infantil” En: Educación XX1. Madrid, España. Vol. 14, núm. 1, 2011, pp. 135-156.

Comisión Intersectorial para la Atención a la Primera Infancia-CIPI (2013). Estrategia de atención integral a la primera infancia-fundamentos políticos, técnicos y de gestión. Bogotá -Colombia.

Comisión Intersectorial para la Atención a la Primera Infancia-CIPI (2011). Fundamentos técnicos de la estrategia de atención integral a la primera infancia. Lineamiento técnico para la protección integral del ejercicio de los derechos de las niñas y los niños desde la primera infancia. Bogotá-Colombia.

Comisión Intersectorial para la Atención a la Primera Infancia-CIPI (2012). Atención integral: prosperidad para la primera infancia-versión actualizada, octubre. Bogotá-Colombia.

Comisión Intersectorial para la Atención a la Primera Infancia-CIPI (2013). Estrategia de atención integral a la primera infancia- fundamentos políticos, técnicos y de gestión. Bogotá -Colombia.

Ministerio de Educación Nacional-MEN (2012). Desarrollo integral en la primera infancia. Modalidades de atención. Comisión intersectorial para la atención de la primera infancia "De cero a siempre". Bogotá -Colombia.

Ministerio de Educación Nacional - MEN (2014). Documento N²0. Sentido de la educación inicial. Bogotá, Panamericana Formas e Impresiones S.A.

Ministerio de Educación Nacional - MEN (2014). Documento $N^{\circ} 19$, Referentes técnicos para la cualificación del talento humano que trabaja con primera infancia. Panamericana Formas e Impresiones S.A. Bogotá-Colombia.

Ministerio de Educación Nacional - MEN (2014). Documento $N^{\circ} 21$. el arte en la educación inicial. Bogotá, Panamericana Formas e Impresiones S.A.

Ministerio Nacional de Educación-MEN (2012). Desarrollo integral en la primera infancia. Modalidades de atención. Comisión intersectorial para la atención de la primera infancia "De cero a siempre" Bogotá.

Ministerio de Educación Nacional - MEN (2014). Documento N 22. El juego en la educación inicial. Panamericana Formas e Impresiones S.A. Bogotá-Colombia.

No. 17. Agosto de 2017 Palobra 
Ministerio de Educación Nacional - MEN (2014). Documento No 23. La literatura en la educación inicial. Panamericana Formas e Impresiones S.A. Bogotá-Colombia.

Ministerio de Educación Nacional - MEN (2014). Documento $\mathrm{N}^{\circ} 24$. La exploración del medio en la educación inicial. Panamericana Formas e Impresiones S.A. Bogotá-Colombia.

Ministerio de Educación Nacional - MEN (2014). Documento $N^{\circ} 25$. Seguimiento al desarrollo integral de las niñas y los niños en la educación inicial. Panamericana Formas e Impresiones S.A. Bogotá-Colombia.

Ministerio de Educación Nacional - MEN (2014). Guía N 50, Modalidades y condiciones de calidad para la educación inicial. Panamericana Formas e Impresiones S.A. Bogotá-Colombia.

Ministerio de Educación Nacional - MEN (2014). Guía N 51. Orientaciones para el cumplimiento de las condiciones de calidad en la modalidad institucional de educación inicial. Panamericana Formas e Impresiones S.A. Bogotá-Colombia.

Ministerio de Educación Nacional - MEN (2014). Guía N 52. Orientaciones para el cumplimiento de las condiciones de calidad en la modalidad familiar de educación inicial. Panamericana Formas e Impresiones S.A. Bogotá-Colombia.

Ministerio de Educación Nacional - MEN (2014). Guía N 53. Guías técnicas para el cumplimiento de las condiciones de calidad en las modalidades de educación inicial. Panamericana Formas e Impresiones S.A. Bogotá-Colombia.

Ministerio de Educación Nacional - MEN (2014). Guía N 54. Guías técnicas para el cumplimiento de las condiciones de calidad en las modalidades de educación inicial. Panamericana Formas e Impresiones S.A. Bogotá-Colombia. 

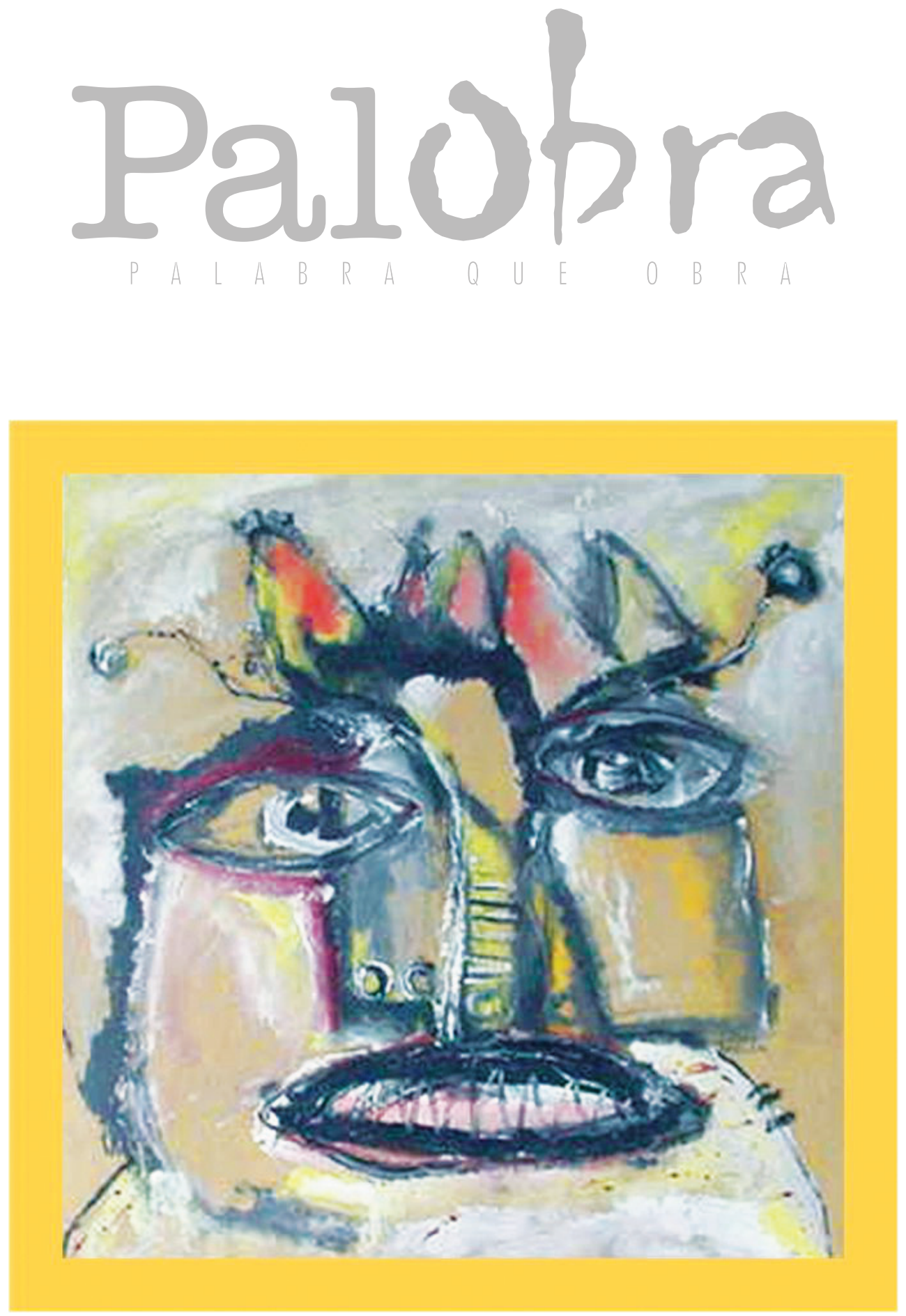

Obra pictórica de la serie "Rostros" de Eduardo Potrillé 